\title{
L'Attention
}

\section{La phénoménologie expérimentale de l'attention de Carl Stumpf}

\section{Natalie Depraz}

\section{(2) OpenEdition}

1 Journals

Édition électronique

URL : http://journals.openedition.org/alter/1728

DOI : $10.4000 /$ alter. 1728

ISSN : 2558-7927

Éditeur :

Association ALTER, Archives Husserl (CNRS-UMR 8547)

\section{Édition imprimée}

Date de publication : 1 octobre 2010

Pagination : 255-274

ISBN : 2-9522374-6-8

ISSN : $1249-8947$

\section{Référence électronique}

Natalie Depraz, «La phénoménologie expérimentale de l'attention de Carl Stumpf », Alter [En ligne], 18| 2010, mis en ligne le 01 juin 2020, consulté le 29 juin 2020. URL : http://journals.openedition.org/ alter/1728; DOI : https://doi.org/10.4000/alter.1728 


\title{
LA PHÉNOMÉNOLOGIE EXPÉRIMENTALE DE L'ATTENTION DE CARL STUMPF
}

\author{
Natalie Depraz
}

La thématique de l'attention apparaît de manière topique dans les deux tomes de la Psychologie du son ${ }^{1}$, publiés respectivement en 1883 et en 1890. W. James, proche ami de Stumpf, publiera de son côté dans The Principles of Psychology (1890) un chapitre entier sur l'attention².

Carl Friedrich Stumpf (1848-1936), psychologue et musicologue, a joué un rôle crucial dans la constitution de la philosophie phénoménologique au tournant du $X x^{e}$ siècle $^{3}$. F. Brentano, qui fut le professeur de Husserl à Vienne, recommanda ce dernier à Stumpf, lui qui fut son premier élève à Würzburg, et qui enseignait alors à Halle. Pendant l'automne 1886, trois années après la publication du premier volume de la Tonpsychologie, Husserl vint préparer son Habilitation (1887) sous sa direction. Il avait également l'intention d'accroître ses connaissances en psychologie et fut impressionné par la façon qu'avait Stumpf de traiter de la relation entre perception et imagination, notamment dans ses cours du semestre 1886-18874. Nous savons aussi que Stumpf, en 1894, encouragea Husserl à lire les Principles of Psychology. Enfin, ce dernier dédie les Recherches logiques (1900-1901) à

1. C. Stumpf, Tonpsychologie, Leipzig, Hirzel, deux volumes, vol. 11883 et vol. 21890 (réimpr. Amsterdam, Bonset, 1965), respectivement $\S 4$ : «Aufmerksamkeit ; Übung ; Ermüdung », p. 67-87 (dont la traduction est proposée ci-après), et $§ 22$ : «Function der Aufmerksamkeit bei der Analyse und dem Heraushören ", p. 276-318, dont je n'ai rendu ici que quelques passages dans la présente présentation, eu égard, notamment, à la longueur du texte (45 p.), lequel paraîtra dans une édition séparée.

2. Cf. la traduction de ce chapitre (vol. 1, chapitre 11, p. 402-458) par A. Voscaroudis dans le présent ouvrage. Dans les Principles of Psychology (Dover, 1890, p. 911), W. James disait de C. Stumpf : il est "the most philosophical and profound of all writers ; and I owe him much."

3. Cf. à ce propos la très substantielle Introduction de D. Fisette à sa traduction de quatre articles de Stumpf, publiée sous le titre Renaissance de la philosophie. Quatre articles, Paris, Vrin, 2006.

4. Le témoignage de Husserl se trouve notamment dans le Ms. F I 8/8a. 


\section{L'attention}

Stumpf en témoignage d'amitié et de reconnaissance de son œuvre philosophique.

Stumpf est par ailleurs le fondateur de l'Institut de Psychologie à Berlin, qui donna naissance à la psychologie de la forme, ce courant majeur de la psychologie avec lequel Husserl fait implicitement alliance tout en marquant ses réserves à l'égard d'une autre psychologie, élémentariste (à savoir sensualiste : atomico-associationniste) ; depuis cet Institut, il a notamment pour élèves W. Köhler, K. Koffka, W. Wertheimer et K. Lewin. Il apparaît donc clairement que la psychologie descriptive est l'un des axes principaux de la philosophie de C. Stumpf. Ce dont témoigne la renommée de la Psychologie du son, largement reçue en Allemagne et en Angleterre (recensée par P. Natorp, A. Meinong, T. Lipps et J. Sully).

L'ouvrage traite des phénomènes liés à la perception des sons et, plus spécifiquement, des jugements provoqués par les sons, et l'auteur y développe quelques concepts importants : l'analyse, l'attention, les relations, et le bien connu concept de "fusion", à la base de sa théorie de la musique (en lien avec la «consonance »). Le premier volume, d'où est tirée la traduction qui suit, traite des jugements portant sur les sons isolés, tandis que le second étudie la conscience de sons simultanés. Au départ, Stumpf devait écrire deux autres volumes, centrés sur les phénomènes musicaux comme la consonance, la dissonance, les chœurs, les mélodies, et le quatrième sur les sentiments engendrés par le son (Tongefühle). Ces questions ont été partiellement publiées dans ses Beiträge zur Akustik und Musikwissenschaft [9 volumes].

La Psychologie du son se situe par ailleurs entre l'ouvrage inaugural de Stumpf, qui date de 1873 (un an avant la Psychologie d'un point de vue empirique de F. Brentano son maître), intitulé Über den psychologischen Ursprung der Raumvorstellung (Leipzig, Hirzel) et consacré à la représentation de l'espace, et les textes plus tardifs (1906), plus méthodologiques et épistémologiques, consacrés à la notion de "fonction " et à la "classification des sciences", voire très tardifs et traitant des émotions et des sentiments $\left(1928^{5}\right)$.

Étant donnée la méticulosité des présentations de la philosophie de Stumpf mentionnées ci-dessus, on peut s'y étonner de la très faible importance qui y est accordée à la Psychologie du son d'une part, et plus particulièrement, de l'absence, si ce n'est à titre de simple mention, d'un compte-rendu détaillé de la conception stumpfienne de l'attention. Aussi allons-nous nous pencher plus particulièrement sur cette conception, pour permettre au lecteur de mieux en évaluer la portée, la possible originalité, et de pouvoir plus précisément la situer par rapport à celle de James, de Titchener ou de Külpe ${ }^{6}$.

5. À propos des textes sur l'espace, sur la fonction, ou sur les émotions, je me permets de renvoyer à la Préface de D. Fisette, op. cit., ainsi qu'à l'article «Carl Stumpf » de Stanford Encyclopedia of Philosophy (Internet), 28 janvier 2009, qui font tous deux droit de façon extrêmement minutieuse à la relation avec Kant, Brentano, Husserl et la psychologie de la forme.

6. Cf. les recensions par A. Flajoliet, dans le présent ouvrage, de Grundriss des Psychologie (1893) de O. Külpe et de Lectures on the Elementary Psychology of Feeling and Attention (1908) de E.B. Titchener. 
En 1883, dans le premier volume de la Psychologie du son, Stumpf décrit l'attention comme un intérêt qui s'éveille à la rencontre d'un objet et correspond à un sentiment : « l'attention est identique à un intérêt et l'intérêt est un sentiment " ( Aufmerksamkeit ist identisch mit Interesse und Interesse ist ein Gefühl $l^{7}$ ). On ne saurait être plus clair: je reviendrai sur le sens d'une telle affirmation radicale et lapidaire ; en 1890, dans le deuxième volume, ce sentiment sera précisé en termes de plaisir (Lust) : l'attention est une activité qui consiste à s'apercevoir de ce qui nous entoure, à noter et à remarquer (bemerken), et cette activité est motivée par le plaisir que l'on y prend; dès lors, les objets non remarqués ne sont par conséquent pas objets de plaisir (Unlust), tandis que les objets remarqués le sont. C'est ce que Stumpf appelle de manière topique " prendre plaisir à s'apercevoir de ce qui nous entoure » («eine Lust am Bemerken »). En outre, tandis que l'analyse du phénomène de l'attention est en 1883 enracinée dans des expérimentations physicophysiologiques (principalement de Fechner, mais pas seulement) liées à l'apprentissage sensoriel, et de l'écoute en particulier, elle se trouve guidée en 1890 par le paradigme de l'écoute des sons musicaux ${ }^{8}$.

Bref, quel que soit le terrain empirique d'ancrage de l'analyse, physicopsychologique ou phonético-musicologique, la motivation cruciale de l'attention est le plaisir et le déplaisir : elle épouse ce faisant le rythme d'une tension focalisée (Spannung) et d'un relâchement ouvert (Lösung), correspondant à une intensité spécifique du sentiment et impliquant en contre-partie une résistance (Widerstand) voire des obstacles (Hemmungen) qui procèdent du sujet, c'est-àdire engendrent processus de distraction ou d'inattention : «Au moment où [...] un objet "attire l'attention sur lui", naît en nous-même un sentiment de plaisir, une résonance, une reproduction du plaisir lié antérieurement aux perceptions spontanées; et ce plaisir pris à remarquer (Lust am Bemerken) conduit alors de son côté à l'activité effective de remarquer dans le nouveau $\operatorname{cas}^{9}$. » Ou encore : «(...) partout où une perception nous procure du plaisir, que le perçu soit agréable ou non, nous parlons d'attention ${ }^{10}$. »

C'est cette thèse forte selon laquelle l'attention reçoit sa motivation principale dans le plaisir que l'on prend à faire quelque chose (Lust am Bemerken) qui fera l'objet d'une discussion critique serrée par Husserl dans son cours de 1904-1905 consacré à l'attention ${ }^{11}$. Je ne re-détaillerai pas ici

\section{Op. cit., t. 1, 1883, p. 68 .}

8. Dès la Préface à l'ouvrage, Stumpf témoigne en guise de motivation personnelle à l'écriture du livre d'un intérêt " passionné " pour la musique, pour laquelle il envisageait alors de faire carrière, sa rencontre avec Brentano et la philosophie ayant encore aiguisé son amour de jeunesse (op. cit., 1883, p. V).

9. Op. cit., t. 2, 1890, p. 282.

10. Op. cit., t. 2, 1890, p. 280. Stumpf se réfère alors en note à l'identité notée dans le volume 1 entre « attention », « intérêt » et « sentiment », en mentionnant le fait que cette identité a suscité des controverses mais aussi des alliances, notamment celle de Ribot.

11. E. Husserl, Hua XXXVIII, Wahrnehmung und Aufmerksamkeit (T. Vongehr et R. Giuliani éd.), Dordrecht, Kluwer, 2004, traduction française par N. Depraz sous le titre Phénoménologie de 


\section{L'attention}

cette discussion, d'autant plus qu'elle trouve son ancrage textuel, chez Stumpf, depuis le $\S 22$ du volume 2 de la Psychologie du son, et non depuis le $\S 4 \mathrm{du}$ volume 1 , lequel seul, à titre de base théorique de la conception stumpfienne de l'attention, a ici retenu mon intérêt. On peut juste noter que, dès 1883, Husserl fait une première critique de l'analyse de l'attention par Stumpf, dite alors vor-phänomenologisch ${ }^{12}$ : en 1890, Husserl substituera au modèle stumpfien de la tension et du relâchement de l'énergie corporelle celui de l'intention et du remplissement, c'est-à-dire l'intentionnalité formelle, refusant ce qu'il interprète comme une description orientée par les contenus (organiques et émotionnels), de façon à promouvoir une description dirigée par les actes (formels) ${ }^{13}$.

Pour nous en tenir à ce stade à la base théorique de 1883, on peut noter que l'analyse de Stumpf met au premier plan l'idée de l'intérêt en tant que sentiment: "Aufmerksamkeit ist Interesse und Interesse ist ein Gefühl ${ }^{14}{ }^{\text {». }}$ Tout le développement souligne le caractère graduel des modes d'attention à la lumière d'exemples concrets tirés des phénomènes du sommeil et de ses contraires ou corrolaires, à savoir l'éveil, le demi-sommeil, la rêverie, ou encore le phénomène de la fatigue et de ses degrés avec la paresse, la concentration, l'entraînement, etc. Stumpf insiste à ce stade sur le processus d'intensification, d'augmentation ou de renforcement (Steigerung, Verstärkung) propre au devenir-attentif et, inversement, sur le processus contraire du devenir-inattentif via la baisse ou la diminution de la force ou de l'intensité. En outre, il analyse les différentes formes d'attention (involontaire, réflexive, contrainte) ainsi que leurs effets. Comme James, qui développe une analyse similaire des degrés sensoriels de l'attention, de ses formes et de ses effets, Stumpf note que le phénomène de l'attention peut être mesuré et analysé parallèlement à un niveau neuro-psychologique, et ce, en s'appuyant sur l'analyse psycho-physique de Fechner, tandis que James s'avère plus proche des expérimentations de Wundt.

Pour développer leur phénoménologie de l'attention, Stumpf (1883) et James (1889) se réfèrent tous deux soigneusement, dans leurs œuvresmaîtresses de ce point de vue, à des données empiriques et à des expérimentations, et ils font usage de leurs résultats à titre de clés de leur analyse. Mais, de façon étonnante, ils procèdent de façon opposée. Stumpf commence par une description expérimentale objective « en troisième

l'attention, Paris, Vrin, 2009. L'Introduction retrace notamment le détail de la discussion avec Stumpf concernant l'attention comme « Lust zum Bemerken » ou non.

12. Manuscrit désormais publié dans le Hua XXXVIII, Appendice II : « Notes sur la doctrine de l'attention et de l'intérêt », p. 159-189, et traduit en français dans E. Husserl, Phénoménologie de l'attention, op. cit.

13. Ниа XXVII, §4. Une telle critique est longuement argumentée dans l'appendice II du Hua XXXVIII, où Stumpf est nommément cité $(\S 1$ : «Début de la discussion avec C. Stumpf : l'attention comme "plaisir pris à remarquer" » (p. 159-160) et $\S 3$ : "Nouvelle discussion critique : exemples à l'encontre de Stumpf », p. 168-171).

14. Op. cit., p. 68. 
personne », dirait-on aujourd'hui dans le contexte du cognitivisme (en prenant appui sur Fechner), et il y adjoint une description des vécus du sujet "en première personne»; à la différence de Stumpf, James donne la primeur à sa propre description personnelle (« en première personne»), et la confirme dans un second temps en mobilisant une perspective objective en troisième personne (qu'il étaye grâce aux expérimentations de Wundt).

Dans le $\S 4$ traduit ci-après, l'auteur procède en trois étapes: tout d'abord, il identifie l'essence de l'attention (p. 68); ensuite, il analyse les causes de l'attention (p. 68-72); enfin, de façon cette fois très détaillée, il étudie ses effets (p. 72-86). Selon Stumpf, on l'a déjà noté à plusieurs reprises, l'essence de l'attention réside dans l'intérêt, lequel se trouve défini de son côté comme un sentiment. Cette définition contient deux corrélats : 1) elle est décisive dans le cadre du sonore/auditif (p. 67) ; 2) l'attention est la condition générale de toute activité interne (p. 68). Partant de là, l'auteur s'intéresse aux causes du phénomène : il insiste tout d'abord sur le rôle des impressions sensibles, ici auditives (en mentionnant aussi la vision). Cependant, il met en question la thèse condillacienne de la "plus grande force » ou de «l'attrait le plus fort » des impressions sensibles, et met en avant, par contraste, l'expérience d'une attention passive involontaire, qui se trouve provoquée par l'apparition d'un objet dans mon champ auditif, ou bien donnée lors d'une lumière plus faible. La force ponctuelle et maximale de l'impression se trouve par conséquent relativisée ici au profit du processus de son accroissement graduel, étayée physiquement par l'exemple empirique, fechnerien, de l'« onde » sonore. En témoignent, à titre d'exemples destinés à rendre compte de l'apparition graduelle et progressive de l'attention, les états de conscience comme la veille, la demi-veille, le sommeil. On comprend pourquoi la première cause évoquée, la force des impressions sensibles, est insuffisante voire unilatérale. Ainsi, après avoir fait droit au côté du contenu de l'objet sensible (en lien avec les causes de l'attention), Stumpf introduit naturellement le côté de l'acte du sujet.

Les composantes de l'acte subjectif de l'émergence de l'attention font partie de ses effets, lesquels forment la troisième étape de l'analyse, la plus détaillée de toutes. On atteint là le niveau de l'étude le plus en relation avec l'exigence d'une phénoménologie expérimentale. Le processus ici central consiste dans un double renforcement ou accroissement (tout à la fois réciproque et corrélatif), à savoir tout à la fois objectif sensible et subjectif vécu. L'expression-clé, ici, est celle de « doppelte Verstärkung 15 ». Stumpf note très bien le caractère circulaire de la conception du phénomène de l'attention: ce double accroissement, tout à la fois sensible et conscient, correspond à la méthodologie d'une causalité circulaire co-générative, dans la mesure où l'accroissement de l'acte subjectif conscient a un effet en retour sur l'accroissement du contenu sensible objectif, lequel fut décrit tout d'abord comme la cause initiale du processus attentionnel. 


\section{L'attention}

De surcroît, ce modèle de la causalité générative permet de décrire le devenir-attentif comme un processus qui comprend un exercice (Übung) dans sa définition : s'exercer à devenir plus attentif, voilà une activité qui fait partie en tant que telle du processus attentionnel. Le rôle du devenirconscient est alors hautement significatif et agit en retour sur la force de l'impression sensible "initiale», c'est-à-dire la renforce, tandis que cet accroissement sensible intensifie de son côté la force du devenir-conscient. Pour étayer empiriquement cette hypothèse philosophique, Stumpf se réfère aux expérimentations de Fechner, qui offrent la possibilité d'une mesure du degré d'intensité de l'attention : on peut accroître une telle intensité s'il y a exercice, et l'on peut également mesurer la diminution de l'attention, lorsque l'on est distrait. De telles expérimentations en troisième personne sont déterminantes : ce sont elles qui vont fournir à Stumpf son concept-clé du renforcement (Verstärkung) ou de l'intensification (Intensifizierung), lequel correspond au premier jalon d'une phénoménologie de l'attention entendue comme attention exercée ${ }^{16}$.

Or, un tel processus d'accroissement de l'attention du fait de son exercice n'est ni linéaire ni téléologique : il a une dimension circulaire de type spiraloïde. En soulignant dans le devenir-attentif l'importance de l'apprentissage, Stumpf distingue à la suite de Fechner un rythme de trois phases différentes, qui correspondent à trois moments spécifiques de l'attentionnalité. Le devenir-attentif est un processus hétérogène, qui comprend ici au moins trois phases différentes : une phase ascendante lente, une phase ascendante rapide, enfin une retombée rapide ${ }^{17}$. L'insistance de Stumpf sur l'exercice en tant que composante essentielle de l'attention, exercice qui permet à cette dernière de se développer, a pour conséquence qu'il ne consacre que peu de pages, en fin de paragraphe, à la fatigue en tant que baisse de l'attention. On peut le regretter, d'autant plus qu'il n'est pas sûr que le phénomène de la fatigue soit le contraire strict et simple de l'exercice, et que celle-ci soit un phénomène simple ou homogène : que dire par exemple de la « lassitude »?

\section{«Attention, exercice, fatigue »}

[67] 1. Parmi les influences que, à propos de chaque classe de jugements, on retrouve dans chaque domaine sensoriel, l'attention tient le premier rang. Mais c'est avec le son, plus qu'avec toute autre modalité sensorielle, que ressort son importance pratique et son intérêt théorique. C'est par les impressions auditives que nous réveillons le dormeur et celui qui rêve éveillé. Un organe toujours ouvert, la pénétration des [68] ondes sonores de tous côtés (on ne peut voir à travers un mur, mais entendre, oui) et de nombreuses autres circonstances sont les causes de cette importance 
pratique. Le fait que l'on privilégie le langage des sons par rapport au langage des gestes en est en partie la conséquence; par ailleurs, le langage des sons, une fois qu' on sait l'utiliser, contribue à son tour à accroître encore l'efficacité psychique des impressions auditives selon l'orientation mentionnée. D'après Schopenhauer, la sensibilité auditive serait pour ainsi dire un critère de la finesse de l'organisation de l'esprit. Aussi est-il naturel que l'attention, dont dépend en dernière instance toute l'activité de l'esprit, déploie pleinement et prioritairement son essence et son effet dans les jugements auditifs, et que la théorie de l'attention y soit le plus développée. Mais, avant de nous engager dans la discussion des phénomènes (ce que nous ferons en effet aux § 15 à 21), il convient aussi d'en dire par avance quelque chose d'un peu plus général ou, du moins, d'identifier les points à interroger.

La recherche générale consacrée à l'attention (abstraction faite pour l'instant de sa mesure) pose la question de son essence, de ses causes et de ses effets. Pour ce qui est de la première, on peut à peine, d'emblée, émettre des doutes à son propos : attention et intérêt sont synonymes, et l'intérêt est un sentiment. En disant cela, on a tout dit. Il n'est pas possible de définir la qualité particulière de ce sentiment, pas plus que celle d'un autre sentiment, comme la colère, la pitié. On peut le décrire à travers les traits qui l'accompagnent constamment, en particulier les causes et les effets; on peut dire entre autres choses qu'il s'agit d'un sentiment théorique, dans la mesure où il se trouve de façon générale dans un rapport causal étroit et privilégié avec la perception, avec la pensée, et dans la mesure où il favorise la fiabilité (Zuverlässigkeit ${ }^{18}$ ) de ces dernières, tandis que d'autres sentiments l'entravent (sauf si, précisément, ils éveillent l'attention). Mais il n'y a là aucune définition de sa qualité particulière, que l'on ne peut qu'expérimenter de l'intérieur, comme l'on ne peut que voir ou sentir la qualité du rouge ou de la chaleur.

Les causes qui suscitent l'attention sont innombrables, et l'on pourra difficilement les ranger complètement dans des classes fixes [69]. Aussi peu que les causes de la colère. Pourtant, avant toute autre chose, chaque modification a des chances d'éveiller l'attention, et ce, d'autant plus que cette modification est importante, tandis que l'attention diminue aussitôt face à des impressions sensibles constantes (à ne pas confondre avec la fatigue de l'organe). Parmi des impressions simultanées, c'est à son tour, toutes choses étant égales par ailleurs, l'impression la plus forte ${ }^{19}$, de même,

18. Le terme est plus couramment traduit par "sûreté, authenticité, véracité »; j'ai cherché, avec «fiabilité ", à rendre compte du segment sémantique «sur qui on peut compter » (zuverlässig), comme un ami "sûr », l'attention accompagnant et favorisant la perception, où "fiable » fait entendre « fier » et se relie à « confiance » (n.d.t.).

19. Condillac, qui reconnut bel et bien l'importance fondamentale de l'attention pour la vie psychique, méconnut pourtant tellement sa nature particulière qu'il l'identifia simplement à la force plus grande d'une impression; confondant même manifestement avec elle les causes, peut-être aussi les effets de l'attention, et omettant par là même entièrement de voir que l'attention est susceptible de se porter vers des impressions plus faibles, que ce soit lorsque l'on 


\section{L'attention}

toutes choses étant égales par ailleurs, c'est la plus agréable, ou bien celle qui reproduit des représentations déjà intéressantes au préalable, ou bien finalement celle qui est à la base d'une volonté quelconque, qui offre l'impulsion la plus forte pour l'attention. Dans ce dernier cas, nous parlons d'attention volontaire. La volonté n'est pas à proprement parler dirigée vers l'attention, mais vers n'importe quelle connaissance (comparaison, discrimination), dont la base forme la représentation correspondante, et qui peut être de son côté la base d'une action. La volonté ne produit donc rien ici, mais elle est l'attention.

Hormis les classes plus générales de causes mentionnées et du sein de ces dernières, des traits particuliers peuvent être nommés à propos de modalités sensorielles individuelles, comme la stimulation des contours dans la vision. On examinera plus en détail ce que peut offrir à ce propos l'audition.

Mais, si l'on entend par ailleurs par «cause» chaque condition dont dépendent la présence ou l'intensité de l'attention, il faut aussi compter, parmi les causes de l'attention, une classe importante, à savoir les états de l'organe central, en particulier [70] la veille, la demi-veille, le sommeil, quoiqu'il existe naturellement un nombre indéfini de degrés. Dans tous ces états apparaissent des appréhensions d'impressions sensibles (même durant le sommeil, la perception sensible n'est pas totalement émoussée), dont la fiabilité très variable s'ancre manifestement dans les différences d'attention. Cependant, non seulement la veille et le sommeil, mais encore la faim et la satisfaction et des milliers d'états indéfinissables de l'organe central relèvent de cela, états qui favorisent ou entravent différemment, selon l'expérience, la croissance volontaire de l'attention. La "vague de fond» (Unterwelle) se situe plus haut ou plus bas, pour parler comme Fechner ${ }^{20}$. Il faudrait

fixe des objets faiblement éclairés à côté d'objets fortement éclairés, ou encore dans le cas des tons harmoniques. L'attention involontaire n'est pas non plus suscitée par la force plus grande d'une impression, un objet peut surgir dans les parties latérales du champ visuel, et éveiller tout autant notre intérêt.

20. On peut bien se représenter avec Fechner (Éléments, II, p. 452 sq.), à maints égards, le rapport entre ces conditions générales et spécifiques d'attention par deux vagues superposées, l'une dotée d'une périodicité très longue et l'autre d'une périodicité très courte, quoique l'on considère l'image seulement comme une image et que l'on n'en conclue rien d'autre que ce que les observations, du reste, nous enseignent. Dans ce cadre, je voudrais défendre Fechner contre le jugement négatif de G.E. Müller, Fechner qui souligna pour la première fois le paradoxe de tant de phénomènes apparentés et chercha à les rassembler dans une seule et même conception, aussi imagée celle-ci soit-elle : «c'est pourquoi nous laissons totalement de côté une critique de ces présentations de Fechner qui prennent entièrement appui sur sa théorie du sommeil et de la veille et qui pour cette raison flottent en l'air, qui sont en partie extrêmement étranges et sont jusqu'ici à peine prises en considération d'une manière ou d'une autre " (Fondement de la psychophysique, p. 359). Exner a abouti à la même image lorsqu'il traitait de l'attention : «Je voudrais ici comparer le degré et le déroulement de l'attention avec une surface flottante (wogenden) que nous pouvons certes soulever dans son ensemble, mais par rapport à laquelle nous n'avons aucune prise s'il s'agit de ses quelques pics. » (Archives de Pflüger, 1875, XI, 429. Voir aussi le Manuel de Hermann, II, 2, p. 286). Donc, même de petits frémissements 
explorer plus avant si cette influence des dispositions organiques sur l'attention est directe ou bien si elle est médiatisée par certaines sensations ou sentiments.

Pour finir, l'attention, eu égard au maximum momentanément atteignable, est dépendante des [71] cas précédents, en l'occurrence, de l'exercice et de la fatigue (pour autant que cette dernière n'est pas comprise parmi les circonstances déjà nommées) ; et elle est certainement également dépendante d'une constante individuelle qui, même si toutes les autres conditions étaient les mêmes pour deux individus, doit correspondre, dans le maximum d'attention atteignable, à une différence dans la capacité à s'apercevoir («l'équation personnelle » repose en partie là-dessus). Néanmoins, il ne semble pas y avoir de différences individuelles dans la capacité $\mathrm{d}$ 'aperception pour des classes particulières de phénomènes sensoriels : une fois rendue présente, la situation devrait pouvoir être, dans une certaine mesure, identique à l'égard d'impressions sensorielles de toutes sortes; et là où elle se manifeste comme factuellement différente, des différences innées concernant l'avantage d'impressions sensorielles diverses en relation avec un exercice différent dans l'aperception devraient suffire à l'explication.

Les questions les plus importantes, surtout pour nos objectifs, concernent les effets de l'attention, étant donné que nous les prenons comme l'un des facteurs qui influent sur la fiabilité du jugement. Avant toute chose, la question est de savoir si par l'attention l'impression correspondante est renforcée. On est tout d'abord porté à faire cette hypothèse, et maints théoriciens en sont également restés là. L'exemple immédiat de la fixation d'un objet vu auparavant de façon indirecte, lequel gagne en puissance lumineuse en vertu de la rotation de l'œil, parle en faveur de cette hypothèse. Mais il est aussi possible, avec un peu d'exercice, de tourner également son attention vers quelque chose que l'on voit de façon indirecte sans bouger les yeux, par exemple lorsque l'on observe une tache aveugle. Et il faudrait pour commencer examiner si un renforcement apparaît ici aussi (dans l'entourage de la tache). Et qu'en est-il si je veux justement porter moimême mon attention sur la force d'une impression? Alors, la force qui est censée être observée s'accroîtrait eo ipso avec l'observation, je ne pourrais donc absolument jamais observer un son faible: je ne pourrais observer qu'un son dont la force est au maximum ou qui, du moins, croît de manière constante durant l'observation; alors que, de fait, je peux même, en mobilisant une attention croissante, suivre un son en train de diminuer. [72] On ne saurait sinon, à propos de jugements portant sur la force, considérer l'attention comme une condition favorisant la fiabilité. Or, on doit peut-être distinguer un double renforcement, celui de l'impression sensible et celui de la conscience: l'effet de l'attention réside-t-il uniquement dans cette dernière ? Le son, dirions-nous, n'est pas plus haut, c'est nous qui en avons

(Kräuselungen) deviennent des grandes vagues de fond, que l'on remarque lors d'un accroissement extrême et involontaire de l'attention. » 


\section{L'attention}

une conscience plus aiguë ${ }^{21}$. Même si une telle distinction peut en un certain sens demeurer opérante, elle est néanmoins contradictoire, telle quelle, avec le principe qui vaut par ailleurs comme fondement, selon lequel la force d'un contenu et la force de l'acte correspondant de la représentation (de la conscience de ce contenu) sont une seule et même chose. L'audition nous paraît d'une grande force si le son est d'une grande force 22 . Avec ces difficultés et d'autres semblables apparaît la circularité de la réflexion, ce qui est fréquemment le cas avec les phénomènes psychiques les plus habituels.

La mention des nombreux faits individuels que, à cet égard, l'écoute propose, conduit à une opinion dont nous allons fournir par avance les traits principaux. Assurément, il y a moyennant l'attention un renforcement d'un phénomène sensible, néanmoins seulement tant que le renforcement a atteint le point qui a été provoqué par la stimulation, si tant est que certaines influences secondaires ne produisent pas des effets contraires dans le système nerveux. On voit que, de cette façon, l'attention favorise la fiabilité objective, y compris relativement au jugement portant sur la force. Mais la fonction essentielle de l'attention ne consiste pas en cela, elle consiste bien plus à conserver plus longtemps la représentation dans la conscience; durant ce temps de conservation, le jugement comparatif a le temps de mettre en rapport de multiples relations de la représentation [73] avec $\mathrm{d}$ 'autres relations. Or, tandis que ces dernières, en plus des représentations de relations, s'associent à la première, celle-ci s'inscrit plus fermement dans la mémoire. Une telle conservation plus longue dans la conscience produit l'attention, mais, semble-t-il, pas simplement par renforcement, mais aussi dans des cas où le renforcement n'intervient pratiquement pas. N'est-ce pas une hypothèse importée arbitrairement de la physique que, même dans le cours de la représentation, seule la force donne l'impulsion ? Peut-être que le «comment» ne peut, dans notre cas, absolument pas être expliqué plus avant. Le processus tel qu'il fut décrit est tel, que, ce qui est appelé concentration de l'attention est plus facilement appréhendable comme une concentration de la conscience via l'attention (des représentations, et des jugements portés sur l'une d'entre elles ou sur des représentations peu liées entre elles), de telle sorte que, par là, la fiabilité subjective est augmentée : moyennant le maintien dans la durée de la représentation objet de jugement, l'influence des autres circonstances, lesquelles déterminent par ailleurs aisément le jugement, diminue (pour autant que ces dernières, en vertu de causes particulières, ne demeurent pas pareillement constantes).

Pour procéder à la mesure de l'attention, deux choses sont requises: premièrement, il faut trouver des moyens qui permettent de connaître le

21. Fechner s'exprime en ces termes dans Questions de psychologie, p. 85-86. Mais, dans la «Révision» (p. 270 sq.), il trouve finalement vraisemblable qu'ait lieu un renforcement imperceptiblement faible du phénomène lui-même, et qu'un renforcement plus important n'ait lieu que dans de rares cas exceptionnels, après de nombreux exercices (Fechner parle d'un cas comme d'un « unicum ».)

22. Voir Brentano, Psychologie d'un point de vue empirique, I, p. 157. 
même degré d'attention dans des cas différents de jugements, car, alors seulement, l'influence d'autres facteurs sur le jugement peut être déterminée exactement ; mais, deuxièmement, il faut trouver des moyens de différencier la gradation de l'attention et, par là même, aussi, de rendre mesurable le changement de fiabilité lors du changement de ces facteurs. Sous un premier rapport, nous ne parvenons pas à une évaluation, laquelle, dans ces circonstances, peut pourtant suffire avec le degré d'exactitude peu élevé des résultats requis dans de telles choses. On a naturellement choisi le maximum d'attention comme ce point où elle peut avoir une valeur de grandeur identique. Or, le maximum que je puis atteindre à un moment donné est différent de celui que je puis atteindre en m'exerçant davantage, et ce, pour un même jugement. Mais le cours de l'exercice (voir plus bas) enseigne que, lorsque le niveau de ce dernier est élevé, la fiabilité [74] du jugement, de fait, ne change plus guère ; par conséquent, aussi, le maximum d'attention qu'un individu donné très exercé peut atteindre peut avoir une validité inchangée. Or, l'individu est de son côté également dépendant de conditions organiques, il n'est pas le même à différentes heures du jour, etc. : à cet égard, nous possédons vis-à-vis de certains sentiments qui accompagnent un effort plus ou moins grand (ainsi que l'on décrit plus précisément ces sentiments qui ne sont pas eux-mêmes identiques à l'attention) un moyen de nous assurer si notre disposition est approximativement la même dans deux cas distincts. Mais, finalement, le maximum atteignable peut encore varier, toutes choses étant égales par ailleurs, entre deux individus : à cet égard, nous pouvons pour ainsi dire présumer en général qu'il s'agit de la même grandeur si l'on part du même degré d'intelligence, mais nous aurons une garantie moins élevée que si les jugements provenaient d'un seul et même individu.

La tâche mentionnée en deuxième lieu fait partie des tâches de la théorie expérimentale des jugements parmi les plus éloignées, et pourtant pas complètement impossibles: il s'agit d'examiner l'influence de l'attention sujette à des variations au même titre que celle d'autres facteurs, par conséquent de constater les modifications de fiabilité qui naissent de la diminution graduelle de l'attention. Le fait qu'on ait commencé récemment à introduire des paramètres qui complexifient la tâche semblent déjà montrer que cela n'est pas tout à fait impossible ${ }^{23}$. On peut, au moment de l'appréciation de phénomènes simples comme la similarité ou la différence de deux sons, avoir en tête des moyens pour produire des gradations de l'attention: on peut avoir égard, simultanément, à d'autres phénomènes simples, lesquels sont susceptibles de se démultiplier à mesure. En mesurant une telle démultiplication, [75] on a alors une mesure non pas directe mais

23. Wundt II, 241 sq. Dans la « Révision » de Fechner (p. 144), je lis également le compte-rendu de Boas concernant les essais d'estimation à vue d'œil, qui sont établis avec des degrés différents d'attention, où l'attention a été distraite par une pièce musicale simultanée ; essais qui donnent une équation constante remarquable de la valeur $E=h . T$ (où $h$ correspond à l'estimation précise, T à la marge d'erreur : la « valeur seuil totale »). 


\section{L'attention}

indirecte de l'attention. On peut mesurer, non pas l'attention elle-même, mais les moyens de la diminuer. Mais on devrait seulement évoquer ces possibilités lointaines, puisque nous ne pouvons en rien contribuer ici à leur réalisation, et qu'il y a une certaine excitation à se représenter, non pas comment elles sont formulables de façon problématique en tant que problèmes, mais, comment elles le sont de façon précise, en relation avec d'autres possibilités scientifiques qui ne nous sont désormais plus tout à fait étrangères.

2. On peut en général entendre par exercice relatif au jugement une disposition acquise, en conséquence de quoi un jugement surgit d'autant plus rapidement et précisément (en même temps d'autant moins vacillant) et en étant doté d'une fiabilité d'autant plus élevée que des jugements semblables ont déjà été prononcés fréquemment par le même individu. Une illusion peut aussi devenir une habitude et, en tant que telle, finir par être invétérée, mais on ne parlera pas dans ce cas d'exercice. Le langage utilisé n'est ainsi pas tout à fait en accord avec l'explication donnée, dans la mesure où, par exercice, on n'entend pas seulement la disposition recherchée, mais également le mouvement de la recherche; or, il convient naturellement, si du moins nous sommes soucieux de l'exactitude des définitions, d'éviter cette ambiguïté. Or, à propos de l'exercice concernant le jugement, on peut distinguer semble-t-il deux composantes à partir desquelles cet exercice se forme différemment dans des cas différents :

a) un exercice d'aptitude à la représentation ou de mobilisation de l'imagination (au sens le plus large du terme, ainsi qu'Aristote l'utilise, ce en quoi il comprend aussi les représentations liées à la mémoire): une disposition propice à certaines représentations, née à la suite de cas similaires antérieurs, qu'il s'agisse de représentations de contenus primaires (qualités, intensités, etc.), ou bien d'intervalles, de relations en général. Nous qualifions cette disposition de d'autant plus propice, d'autant plus vive, plus précise, perdurant d'autant plus que les représentations apparaissent dans des circonstances conscientes par ailleurs semblables; ou bien elles apparaissent avec une vivacité semblable dans des circonstances à chaque fois un peu différentes etc. [75] On a déjà souvent évoqué le fait que la mémoire possède une signification inégale pour des classes différentes de jugements; elle a la plus grande importance pour les jugements portant sur des qualités absolues, des hauteurs sonores, des nuances de couleurs, ou bien pour les jugements portant sur des distances absolues, des grandeurs spatiales, des grandeurs temporelles, en général, donc, pour des jugements de qualification. S'il y a un temps semblable entre l'occurrence précédente et l'occurrence actuelle, la fiabilité de tels jugements dépend encore de façon essentielle de la mémoire. Aussi est-ce cette classe de jugements qui est également la plus appropriée pour mesurer une mémoire disponible.

Pourtant, on n'a absolument pas besoin de s'intéresser à la même chose à propos de jugements portant sur des différences tout aussi remarquables. La différence de la fonction de la mémoire à propos des jugements de la classe 1 
et de la classe 2 est pour l'essentiel la suivante : à propos de la première classe (dont fait partie le jugement de qualification), il s'agit en général de reproduire une représentation d'un son par exemple après un intervalle de temps assez long, en quoi cependant un certain espace de jeu d'inexactitude perdure, dont la portée est bien supérieure à la sensibilité à la différence ; par exemple, cela n'a pas d'importance qu'un son donné et à nommer, lequel me rappelle un son précédent reconnu sous ce nom ou bien ce nom lui-même, se distingue simplement tout au plus du son entendu autrefois, pour autant que je le remarquerais si les deux sons étaient donnés immédiatement l'un après l'autre: c'est suffisant si le son donné est davantage semblable au précédent que n'importe quel son de l'échelle - le jugement sera alors correct. Le son peut alors varier $\mathrm{d}^{\prime} \mathrm{un}$ huitième de ton du son précédent, auquel je le fais à présent s'équivaloir sans remarquer la différence; la mémoire peut ici sans dommages, pour un tel intervalle, être déficiente. Par contre, pour des jugements de deuxième classe, il s'agit en règle générale, après un temps très bref, le plus souvent immédiatement après, de reproduire un contenu de sensation avec la plus grande exactitude possible. Comme l'impression n'était absolument pas encore oubliée au sens étroit du terme, la différence de sensation fournit le point de départ de la fiabilité du jugement. Mais, le plus souvent, pourtant, la reproduction a lieu rapidement, l'impression tout juste passée est tirée de la partie latérale du champ visuel de l'esprit et amenée vers son milieu, [77] arrachée à l'arrièreplan de la conscience et confrontée à l'impression actuelle. Et, dans cette mesure, l'exactitude de la mémoire est dès lors également ici essentielle.

La perfection de la mémoire ou le fait de s'exercer à se représenter (par exercice étant toujours entendu le résultat, non le processus) dépend d'un facteur individuel, en dépit du nombre identique de cas précédents et de circonstances par ailleurs semblables. Ce facteur peut à vrai dire, d'un point de vue psychologique, être encore décrit de plus près, il réside en fait dans l'intensité des sentiments qui accompagnent les représentations, mais il ne semble pas tout à fait constant, il est susceptible, bien plus, d'être modifié par des hasards pathologiques et par une mutation lente de la matière centrale, aussi bien en un sens favorable que défavorable. Mais il y a aussi, vraisemblablement, des dispositions variables, d'une part en faveur d'une mémoire à longue terme mais peu précise, d'autre part en faveur d'une mémoire à court terme mais précise, par quoi, alors, $\mathrm{d}$ 'après ce qui vient aussi d'être dit, la disposition à juger selon la première et la deuxième classe serait différente selon l'individu.

Il est jusqu'à présent impossible d'expliquer plus avant l'essence du fait de s'exercer à se représenter. Des psychologues pénétrants comme Lotze ont assez souvent souligné que nous n'avons aucun aperçu du «comment » de l'association et de la reproduction, de l'essence du processus qui arrache une représentation à l'oubli et qui augmente elle-même en puissance moyennant la répétition croissante des cas. Les physiologues seront peut-être plus heureux en cette matière. Jusqu'à présent, ce qui a été dit d'Aristote à nos 


\section{L'attention}

jours sur la démonstration physique de la mémoire n'est en réalité qu'une traduction du connu et du certain en du moins connu et de l'hypothétique, une description dans une langue encore complètement inaccessible à la multitude des faits psychiques à décrire, une imagination de l'imagination, un souvenir du souvenir.

b) S'exercer à être attentif. Une fiabilité considérablement croissante se manifeste aussi fréquemment dans une série de jugements, dans des cas où la mémoire co-opère comparativement moins et où, par là même, un exercice efficient de celle-ci ne peut pas non plus avoir lieu ; il en va ainsi, en effet, de [78] jugements portant sur des différences tout aussi remarquables ${ }^{24}$. On peut attribuer sans scrupule le succès d'un exercice à l'attention, d'autant plus que le progrès éventuel de la mémoire n'est dans ces cas-là qu'un effet de l'attention, car la précision de la reproduction dont il s'agit est justement pour l'essentiel conditionnée par la mémoire. Un son peut au bout d'un certain temps s'imprégner en moi, si je l'entends longtemps ou très souvent mais sans y prêter particulièrement attention, à ceci près que la fidélité de la reproduction n'est de loin pas aussi importante que si je l'ai écoutée avec attention une seule et unique fois, auquel cas néanmoins la durée de la mémoire devrait être moins importante. L'augmentation de la fiabilité des jugements de deuxième classe repose en dernière instance sur la croissance de l'attention. Même si celui qui juge croit avoir déjà porté au tout début la plus grande attention, et est effectivement en possession du maximum qu'il pense pouvoir atteindre, ce maximum peut justement être modifié par de nombreuses circonstances. Il est d'emblée prévisible que ce maximum se déplace avec l'exercice. Car tout sentiment gagne en intensité en se répétant et, par là, en influence sur la vie psychique, pour autant que la répétition ne met pas en même temps en jeu des forces contraires. Parfois on peut obtenir confirmation, à travers les assertions de personnes interrogées, du fait que le jugement s'affranchit toujours plus d'influences secondaires, lesquelles peuvent être identifiées nommément, et se concentre elle-même sur des contenus comparatifs, ce qui, d'après ce qui a été noté plus haut, doit être considéré comme une conséquence de la croissance attentionnelle. Par là, s'explique aussi sans doute le fait que, là où l'on peut repérer des erreurs constantes et variables (§3), le succès de l'exercice tient manifestement à une diminution des erreurs variable ${ }^{25}$. Car l'attention ne diminue [79] l'influence des circonstances secondaires que dans la mesure où celles-ci ne demeurent pas elles-mêmes constantes.

3. Il convient à présent d'aborder certains points qui concernent l'exercice du jugement en général, même si ceux-ci sont à porter principalement au compte de la croissance attentionnelle ou mémorielle. Il s'agit avant tout du

24. A.W. Volkmann (et Fechner). A propos de l'influence de l'exercice sur la connaissance des distances spatiales (lors du toucher). Rapports sur les débats de la Société saxonne des sciences physiques et mathématiques. Cl. X, Bd. 1858, p. 38-69.

25. A.W. Volkmann, « Über das Vermögen, Grössenverhältnisse zu schätzen ». Dans le rapport déjà mentionné, p. 173 et p. 200 sq. 
cours général de l'exercice, du taux de croissance, lequel inclut le nombre croissant des cas, la forme de la courbe de l'exercice. Dans presque toutes les recherches où les auteurs ont porté leur attention là-dessus, il s'est avéré que l'exercice a acquis une valeur beaucoup plus rapidement qu'on ne pouvait le pressentir. Les auteurs manifestent à ce propos leur étonnement de façon quasi unanime ${ }^{26}$. Cependant, la vitesse de l'accroissement est différente pour les jugements de différentes classes. Elle doit en général être plus importante dans le cas où le jugement est soumis à des conditions multiples et dans une large mesure variables dans la fiabilité subjective. Mais cette vitesse considérable de l'exercice semble n'être [80] présente qu'à un certain stade intermédiaire d'exercice. Après qu'un degré élevé de fiabilité est atteint, l'exercice augmente moins rapidement, la courbe prend une forme asymptotique. Dans le cas de sujets très exercés, on ne voit quasiment plus l'effet d'une répétition prolongée. Ceci, qui ressort déjà clairement des expériences de la vie quotidienne, Volkmann a pu le noter dans des essais qui portent directement sur ce point. Dans la plupart des objectifs théoriques et pratiques, on peut sans dommages considérer cet état ultime qui n'est plus variable qu'asymptotiquement comme un maximum constant de l'exercice; ce qui est assurément significatif pour pouvoir conclure à des différences de sensibilité $(\S 3)$. Mais les expériences quotidiennes montrent en outre que, dans tous les domaines, le premier démarrage de l'exercice n'amène tout autant que des progrès lents, quasiment imperceptibles: "tout démarrage est difficile". Ainsi, Fechner, en faisant des essais sur le cours de l'exercice musculaire, lequel ne peut assurément être comparé sans plus avec l'exercice du jugement, a mis au jour une courbe qui croît tout d'abord

26. À ce propos du domaine acoustique, voir Auerbach et Kries, au § 11 [de la Psychologie du son. Non traduit ici. N.d.t.]. Voir Preyer, Limites de la perception sonore, p 30, p. 37 («étonnamment rapide»). J'ai été fréquemment frappé par la même chose lors des essais de non-musiciens (§14). Dans les essais de Volkmann, mentionnés juste avant, concernant la possibilité de la connaissance de différences cutanées très infimes, les différences les plus infimes découvertes par E.H. Weber dans différentes régions de la peau ont été moyennant exercice réduites avec une "rapidité absolument surprenante». Après quelques heures seulement, la finesse de ce sens (de ce jugement) avait à peu près doublé, chez Volkmann lui-même elle avait même quadruplé au niveau du creux de la main. Même dans ses essais consacrés à l'appréciation oculaire des segments brisés d'une grandeur donnée, Volkmann fait mention d'un exercice considérable (art. cit., p. 202). Il en va de même avec Fechner pour les jugements d'identité concernant les différences cutanées (Éléments I, p. 132). Dans les essais de Dobrowolsky et Gaine sur l'acuité visuelle à la périphérie de la rétine (Archives de Pflüger, 1876, p. 411, 420, 427 sq.), on a pu remarquer par moments un exercice manifeste, même après le premier essai. De même, il y a une influence significative de l'exercice dans les essais de M. Friedrich sur la durée d'aperception dans la perception de tableaux de chiffres (Wundt, Études philosophiques, 1881, Vol. 1, Cahier 1, p. 39 sq., p. 68). Les temps de réaction apparaissent en général, dans une large mesure, dépendants de l'exercice. Dans un essai, Exner a trouvé qu'ils étaient divisés par cinq ou six en l'espace de six mois. 


\section{L'attention}

lentement, puis plus rapidement, enfin à nouveau lentement ${ }^{27}$. Dans le cas de jugements portant sur les sens, ce stade est à peine plus fréquemment contrôlable, dans la mesure où, dans la plupart des jugements portant sur les sens, l'âge adulte, en conséquence de l'usage habituel des sens, est déjà dépassé, d'où l'accroissement rapide immédiat au début des essais systématiques. Parfois, comme lors de la connaissance de toutes petites distances au moyen des yeux, on voit déjà apparaître le troisième stade 28 .

On perd la capacité de s'exercer aussi vite qu'on l'a acquise, dès lors qu'il n'y a plus de nouvelles occasions de s'exercer: lorsque l'exercice a été développé au plus haut point, on le perd lentement, jusqu'à paraître impossible à perdre et, de fait, il ne disparaît jamais dans les limbes ; on perd rapidement l'exercice qui en est resté au deuxième stade, [81] lentement celui qui en est au premier stade. Ce qu'il semble du moins d'après les expériences de la vie quotidienne, tout autant dans le domaine musculaire que dans celui des sens; et pour autant que j'y ai porté mon attention dans le domaine acoustique spécifique à diverses occasions. (Cf. par exemple $\S 13$ et $\S 14$.) On trouve à ce propos des essais systématiques chez Volkmann, qui découvrit combien, après six mois de pause, la différence d'augmentation très rapide des sensations tactiles était considérablement retombée ${ }^{29}$.

En dehors du cours de l'exercice, le co-exercice (Mitübung) est d'un grand intérêt, le transfert qui a lieu de lui-même de l'exercice d'une région d'un sens à l'autre, voire d'un sens lui-même à un autre; on peut comparer la croissance corrélative des membres (Darwin). Le co-exercice a d'abord été observé dans le domaine musculaire. E.H. Weber rapporte le fait que son fils, qui avait appris à écrire de la main droite, se découvrit au même moment une disposition significative de la main gauche pour des mouvements symétriques. Les mots écrits de cette main gauche apparaissaient, vus dans le miroir, presqu'aussi bien écrits que ceux de la main droite ${ }^{30}$. Là où, à

27. Rapports sur les débats de la Société saxonne des sciences physiques et mathématiques. Cl. 1857, p. 113. De plus, la courbe avait également la particularité, liée à la fatigue, que la croissance générale était entrecoupée de petites diminutions.

28. A.W. Volkmann, A propos de l'influence de l'exercice (où, à côté du toucher, on examine aussi l'œil), p. 55.

29. Par exemple, une note à propos de la perte complète de l'exercice musculaire chez Fechner, Éléments, I, p. 310.

30. Fechner, "Observations qui semblent probantes, etc. ». Rapports sur les débats de la Société saxonne des sciences physiques et mathématiques. Cl. 1858, p. 70. Comme je racontais par hasard la chose à ma femme, elle nota qu'elle avait souvent constaté la même chose enfant, et écrivit aussitôt une phrase de cette façon avec beaucoup de célérité et de perfection. Depuis cette époque-là, elle ne s'y était pas exercée et ne s'était pas non plus alors appliquée pour ainsi dire à un tel exercice. Elle avait cru devoir rejeter la question de savoir si, relativement à ses expériences liées aux cours de piano qu'elle donna pendant de nombreuses années à l'Académie supérieure de musique de Berlin, l'une des mains avait un effet d'entraînement (mitgeübt) notable sur l'autre. La main gauche s'avéra au début plus agile, plus tard la main droite fit de gros progrès ; mais elle n'avait pas remarqué d'influence réciproque. Il y en a probablement aussi une, d'une certaine manière ; mais ce qui est principalement souligné, c'est à l'évidence ici le perfectionnement 
propos des jugements portant sur les sens, [82] apparaît un co-exercice, il peut principalement provenir d'une croissance parallèle de la mémoire ou de l'attention. En ce qui concerne tout d'abord la mémoire, on peut sans doute appliquer de manière générale certaines techniques de mémorisation, et les transférer par conséquent du domaine où elles ont tout d'abord été par hasard testées à d'autres domaines ; par ailleurs, la nouveauté du matériau peut être gênante. Puis, on estime d'une certaine manière a priori le degré de co-exercice qui doit intervenir dans différents cas où la mémoire joue un rôle important. Cependant, il faudrait faire des essais. On a quelques notations provenant du domaine acoustique (cf. § 14). Pour les perceptions spatiales, on peut très peu transférer les expériences d'une partie de la rétine sur les autres parties ${ }^{31}$, ce qui est manifestement lié à sa sensibilité très inégale en fonction de tous les objets, à savoir la nouveauté relative des formes, des couleurs etc. Mais cela dépendra également si l'on s'est habitué à juger de façon immédiate ou si l'on est par exemple dépendant des sensations musculaires lorsqu'on chante de façon à reproduire une représentation sonore déterminée. Dans ce dernier cas, le co-exercice ne peut sans doute intervenir pour des régions plus hautes ou plus basses pour lesquelles n'existent précisément aucune sensation musculaire du larynx, là où un exercice déjà réalisé pourrait opérer dans la région intermédiaire. Mais dans le premier cas, la mémoire augmente nécessairement plus vite dans la nouvelle région acoustique que dans la région initiale.

Les expériences de Volkmann sont assurément à référer de façon essentielle à un co-exercice de l'attention: en effet, relativement à des jugements portés sur des différences tactiles tout à fait comparables à six endroits différents du côté corporel droit, c'est non seulement la sensibilité de ces endroits qui a doublé, mais simultanément celle des endroits correspondants de la moitié corporelle gauche. [83] À un moindre degré, on a trouvé un co-exercice dans les endroits correspondants des parties voisines de la peau. Par contre, l'exercice de l'extrémité du doigt gauche n'eut aucune influence manifeste d'aucune sorte sur la sensibilité à l'avant-bras gauche. L'œil possède tout aussi peu l'avantage sur l'exercice du jugement cutané. On peut comprendre ces rapports ainsi : chaque type d'exercice a d'autant plus de valeur que les circonstances du cas d'application sont semblables à celles des cas précédents dans lesquels il a été acquis. Lors d'un effort volontaire semblable de l'observateur exercé, l'attention atteint alors pourtant, dans le cas précis, un maximum distinct en fonction de la similarité des circonstances; en particulier, elle est distraite par le nouveau

de l'organe externe relativement à «l'indépendance » des différents doigts, la séparation des mouvements, laquelle, étant donné le mécanisme dystéléologique de la main pour le piano, doit principalement se mettre en place par des transformations structurelles de cet organe ; tandis que, pour l'activité d'écriture, cela dépend davantage de la façon dont l'imagination développe des formes visuelles et des représentations musculaires.

31. Hering (Manuel de Hermann III, 1., p. 571) : «La faculté de reproduction est localisée. » 


\section{L'attention}

en soi et pour soi, déjà en proportion de la nouveauté. Or, pour des parties homologues, les représentations adjacentes et les sentiments sont extrêmement proches (par exemple, le membre correspondant est représenté en sombre, sa situation dans l'espace central, ses sensations musculaires, s'il est capable de mouvement) ; pour des parties voisines, c'est déjà moins le cas, pour des parties éloignées et non homologues, encore moins, pour des sensations de sens différents, c'est minimal. D'où un succès inégal. C'est pourquoi, on peut aussi en tirer la conclusion pour les jugements acoustiques que l'exercice du jugement acquis pour une oreille relativement à différents sons ou à différentes régions sonores opère également de façon correspondante pour l'autre oreille, plus avant, que l'exercice du jugement acquis pour un son ou pour une région sonore opère aussi pour le son voisin ou la région voisine dans la même oreille. Les circonstances adjacentes correspondantes plus ou moins semblables jouent ici de façon privilégiée dans les sentiments qui les accompagnent. Mais, bien entendu, comme pour la mémoire, certaines techniques que l'on peut appliquer de façon générale ou bien des maximes de conduite que l'on a acquises entrent en considération également pour l'exercice de l'attention, et ce, au-delà de la ressemblance des circonstances. La capacité de concentration sur certains points problématiques, l'habitude de se laisser soi-même déterminer seulement par les contenus qui ont la portée d'un jugement, doit, quel que soit le domaine où elle est acquise, être également efficace dans tous les autres. Simplement, là où un exercice conséquent est déjà à l'œuvre [84], une telle influence autre ne sera naturellement plus notable, ce pourquoi un tel transfert d'exercice n'a pas été noté dans les essais mentionnés de la peau à l'œil.

À partir du phénomène du co-exercice, Volkmann a tiré la conséquence concluante selon laquelle l'exercice, pour ce qui est des jugements portant sur les sens, n'a pas son point d'application et son siège dans les organes externes $^{32}$. Formulé autrement : l'exercice ne modifie pas la sensibilité, laquelle est bel et bien conditionnée au premier chef par l'organe. Du moins, ce que nous avons appris à identifier jusqu'à présent comme un exercice dans les séries d'essais ne peut être appréhendé comme une modification de l'organe. Mais la rapidité de la croissance de l'exercice dans sa période intermédiaire conduit également à la même conclusion. Car il n'est pas possible de croire que l'organe puisse connaître en quelques heures une mutation anatomique de taille, s'affiner de moitié ; et il faudrait en ce cas faire l'hypothèse d'une mutation anatomique. On peut aisément provoquer une augmentation de la différence de sensibilité moyennant une modification effective de l'organe pour le sens tactile cutané en râclant l'épiderme au bout des doigts. Mais moyennant exercice, l'épiderme devient plutôt plus épais. Il faudrait donc pour ainsi dire couper et multiplier les terminaisons nerveuses pendant les quelques heures du temps d'exercice, ce qui ne concorde pas avec les autres

32. Hypothèse qui se trouve pourtant souvent exprimée encore de façon directe ou indirecte. Par exemple, cf. Binet, dans la Revue philosophique de Ribot, 1880, p. 293. 
expériences portant sur les mutations organiques et, surtout, ne ramènerait pas aussi vite les choses à la normale après l'arrêt de l'exercice, comme c'est le cas, de fait, quand l'exercice a eu lieu. Il demeure certainement pensable que les processus centraux qui sont liés à la comparaison attentive des sensations, exercent eux-mêmes, peu à peu, un effet rétroactif sur l'organe, il est pensable que des modifications moléculaires de ce dernier aient lieu, analogues à ce que l'on peut présumer dans la structure du violon lorsqu'il «répond » toujours mieux à l'usage, sans que l'on [85] puisse trouver une modification visible. Même le darwinisme inclut l'hypothèse de telles modifications, qui sont co-conditionnées par l'usage de l'organe; mais, pour les organes des sens, elles pourraient n'être précisément que minimales dans la vie de l'individu. Ce que nous pouvons mesurer en termes de résultat de l'exercice sur un temps court ne saurait se rapporter à cela.

4. Quelques mots, pour finir, à propos de l'opposé de l'exercice, la fatigue. Il n'y a pour commencer pas grand-chose à en dire. Elle se manifeste à partir de deux des conditions du jugement: la sensation et l'attention. Au premier abord, après un très long moment ou après la répétition le plus souvent ininterrompue de l'essai, la force de l'impression diminue et, en lien avec elle, bien souvent, la durée ; et, dans certains cas, il y a en même temps un changement qualitatif. Jusqu'à présent, on n'a procédé à des recherches sur le processus de la fatigue que sur quelques modalités sensorielles. Elle est proportionnelle à la force et à la durée de l'excitation, mais, en même temps, elle est dépendante, tout autant, de modifications de l'organe et des nerfs (y compris des terminaisons nerveuses). Ce sont les expériences de Fechner, étendues à la "loi du parallèle", qui correspondent aux faits les plus généraux, quoiqu'ils ne soient en rien généraux, et d'après lesquelles deux sensations d'un organe produites par une excitation inégalement forte diminuent sous l'effet de la fatigue, de sorte que nous avons l'impression qu'elles sont pour ainsi dire égales en force (essais sur l'égalité des différences de luminosité sur la différence entre deux poids ${ }^{33}$ ). Le degré de fatigue, la rapidité avec laquelle une sensation connaît de la fatigue diffère selon les modalités sensorielles: dans le cas de l'ouie, elle n'est que minimale.

La fatigue attentionnelle entre d'autant plus en considération, de façon privilégiée l'attention involontaire, laquelle connaît une fatigue rapide lorsque des bruits uniformes perdurent très longtemps et a pour conséquence un émoussement des différenciations dans la masse des multiples sensations simultanées et indifférenciées. Mais l'attention volontaire elle-même, qui est présupposée dans les jugements à visée scientifique, connaît la fatigue, et ce, de façon d'autant plus rapide et totale qu'un très gros effort de la volonté a été nécessaire; d'où une fatigue impressionnante dans les essais de

33. Fechner, «Révision», p. 143, p. 181. Les observations de Kries sur ce plan sont à rapprocher de celles de Fechner : deux lumières qui ont l'air semblables apparaissent telles lorsqu'elles sont appréhendées sous l'effet de la fatigue («Les sensations faciales », 1882, p. 109). 


\section{L'attention}

perception possible de toutes petites différences ${ }^{34}$. L'entrée en scène de la fatigue doit souvent, dans des séries d'essais, être clairement contrôlée à un moment déterminé. Mais pourquoi et de quelle manière une fatigue attentionnelle apparaît en général, pourquoi l'attention n'est pas aussi puissante après le centième cas, étant donnée qu'elle est plus puissante en vertu de l'exercice que lors du premier cas : on ne dispose à l'heure actuelle $\mathrm{d}^{\prime}$ aucune hypothèse pertinente concernant ce fait qui domine l'ensemble de la vie psychique, encore moins qu'à propos de la raison de la fatigue des sens ; quoique l'on puisse naturellement faire l'hypothèse en général que les deux faits sont liés au besoin nutritif du complexe nerveux central et périphérique.

La détente du nerf est conditionnée par le type et le degré de fatigue ${ }^{35}$. Elle augmente, semble-t-il, tout d'abord lentement, puis rapidement, finalement, à nouveau encore lentement, jusqu'à ce que l'état normal soit atteint; de même, les processus de détente de l'ensemble de l'organisme se déroulent aussi, habituellement, de la même manière ${ }^{36}$.

Peut-être cette courbe donne-t-elle de façon générale quelques points d'appui pour explorer des processus qui sous-tendent communément tous ces phénomènes, étant donnée qu'on la trouve semblablement pour l'exercice et qu'elle n'est pas moins observée lors de la convulsion d'un muscle, à ceci près qu'elle se répartit sur des surfaces temporelles (en abscisse) très petites ou très grandes.

34. Cf. les remarques d'Exner concernant ses essais à propos des différences temporelles infimes entre sensations, Archives de Pflüger, XI, p. 428-429.

35. J. Bernstein, Archives de Pflüger, XV (1877), p. 295, 302, 318

36. Comparer avec la régénérescence de la rétine après des stimulations lumineuses dans des diverses circonstances de stimulation. M. Peschel, Archives de Pflüger, XX (1880), p. 425. 